\title{
Association of LOXL1 Common Sequence Variants in German and Italian Patients with Pseudoexfoliation Syndrome and Pseudoexfoliation Glaucoma
}

\author{
Francesca Pasutto, ${ }^{1}$ Mandy Krumbiegel, ${ }^{1}$ Christian Y. Mardin, ${ }^{2}$ Daniela Paoli, ${ }^{3}$ \\ Robert Lämmer, ${ }^{2}$ Bernhard H. F. Weber, ${ }^{4}$ Friedrich E. Kruse, ${ }^{2}$ Ursula Schlötzer-Schrehardt, ${ }^{2}$ \\ and André Reis ${ }^{1}$
}

Purpose. Three common sequence variants in the lysyl oxidaselike 1 ( $L O X L 1)$ gene were recently associated with both pseudoexfoliation (PEX) and pseudoexfoliation glaucoma (PEXG) in populations from Iceland and Sweden. In this study, the genetic association of these variants was investigated in patients with PEX or PEXG of German and Italian descent.

Methods. The three LOXL1 single-nucleotide polymorphisms (SNPs), one intronic (rs2165241) and two nonsynonymous coding SNPs (rs1048661: R141L and rs3825942: G153D) were genotyped in a total of 726 unrelated patients with PEX or PEXG (517 Germans and 209 Italians) and 418 healthy subjects who had normal findings in repeated ophthalmic examinations, and a genetic association study was performed.

RESUlTs. Strong association with the three LOXL1 common sequence variants was seen in both the PEX and PEXG patient groups independent of their geographic origin (rs2165241, combined $\mathrm{OR}=3.42, P=1.28 \times 10^{-40} ;$ rs1048661, OR $=$ $2.43, P=2.90 \times 10^{-19}$; and $\mathrm{rs} 3825942$, OR $=4.87, P=$ $\left.8.22 \times 10^{-23}\right)$. Similarly, the common frequent haplotype (G-G) composed of the two coding SNPs (rs1048661 and rs3825942) was strongly associated in PEX and PEXG cohorts of both populations with the disease (combined OR $=3.58$, $P=5.21 \times 10^{-43}$ ).

Conclusions. Genetic variants in $L O X L 1$ confer risk to PEX in German and Italian populations, independent of the presence of secondary glaucoma, confirming findings in patients from Northern Europe. (Invest Ophthalmol Vis Sci. 2008;49: 1459-1463) DOI:10.1167/iovs.07-1449

$\mathrm{P}$ seudoexfoliation glaucoma (PEXG) is the most common identifiable cause of open-angle glaucoma, accounting for approximately $25 \%$ of all open-angle glaucoma worldwide and most cases of glaucoma in some countries. ${ }^{1}$ For instance, it accounts for $77 \%$ of all open-angle glaucoma in the eastern

From the ${ }^{1}$ Institute of Human Genetics and the ${ }^{2}$ Department of Ophthalmology, Friedrich-Alexander-University Erlangen-Nuremberg, Erlangen, Germany; the ${ }^{3}$ Azienda Ospedaliera di Monfalcone, Reparto di Oftalmologia, Monfalcone, Italy; and the ${ }^{4}$ Institute of Human Genetics, University of Regensburg, Regensburg, Germany.

Supported by Grant SFB 539 and, in part, WE1259/14-3 from the German Research Foundation (DFG).

Submitted for publication November 11, 2007; revised December 20, 2007; accepted February 15, 2008.

Disclosure: F. Pasutto, None; M. Krumbiegel, None; C.Y. Mardin, None; D. Paoli, None; R. Lämmer, None; B.H.F. Weber, None; F.E. Kruse, None; U. Schlötzer-Schrehardt, None; A. Reis, None

The publication costs of this article were defrayed in part by page charge payment. This article must therefore be marked "advertise$m e n t "$ in accordance with 18 U.S.C. $\$ 1734$ solely to indicate this fact.

Corresponding author: André Reis, Institute of Human Genetics, Friedrich-Alexander-University Erlangen-Nuremberg, Schwabachanlage 10, 91054 Erlangen, Germany; reis@humgenet.uni-erlangen.de. region of the Arabian peninsula. ${ }^{2}$ Compared with primary open-angle glaucoma (POAG), PEXG has a more serious clinical course, a more rapid progression, and a worse prognosis and is more difficult to treat. ${ }^{3}$

The underlying disorder, PEX syndrome, is an age-related systemic disease of the extracellular matrix characterized by the multifocal production and progressive accumulation of a fibrillar extracellular material in intra- and extraocular tissues that is either the result of an excessive production or insufficient breakdown or both. ${ }^{4}$ Active involvement of the trabecular meshwork in this matrix process may lead to glaucoma development in about half of patients with PEX. Although its exact etiology and pathogenesis are still unknown, recent molecular biological and biochemical data support the pathogenetic concept of PEX syndrome as a type of stress-induced elastic microfibrillopathy, associated with the excessive production and abnormal aggregation of elastic microfibrils by a variety of potentially elastogenic cell types. ${ }^{5,6}$ Although a cause-and-effect relationship of PEX and other systemic diseases has not been established, increasing evidence suggests that PEX syndrome is associated with cardiovascular and cerebrovascular diseases. ${ }^{6,7}$

PEX syndrome occurs in all geographic regions worldwide, with reported prevalence rates averaging approximately $10 \%$ to $20 \%$ of the general population over age $60 .{ }^{8}$ Several lines of evidence show the tendency for the condition to cluster geographically and in certain racial or ethnic subgroups in difference prevalence, ${ }^{9}$ family aggregation and an increased risk of PEX in relatives of affected subjects suggest underlying genetic factors that predispose to this condition. ${ }^{10,11}$

Recently, a genome-wide association study detected three common SNPs on chromosome 15, area q24.1, in the lysyl oxidase-like 1 ( $L O X L 1)$ gene, which is associated both with PEX and PEXG in Icelandic and Swedish populations. ${ }^{12}$ Strikingly, the results indicate that these gene polymorphisms are major susceptibility variants for PEX and support the notion that they confer risk of glaucoma mainly through PEX, as no association was observed in glaucoma patients only. Moreover, the disease-associated polymorphisms appeared to be present in virtually all patients with PEXG within the populations studied. ${ }^{12}$

The product of $L O X L 1$ is a member of the lysyl oxidase (LO) protein family involved in the cross-linking of collagen and elastin in the extracellular space thereby stabilizing and insolubilizing polymeric elastin and collagen. It is required for elastic tissue homeostasis and is ubiquitously expressed. ${ }^{13,14}$ LOXL1deficient mice showed reduced elastin content in different main organs and also had pelvic organ prolapse, enlarged airspaces of the lung, and skin and vascular abnormalities, but to our knowledge no eye phenotype was reported. ${ }^{15}$ Although LOXL1 is responsible for PEX in non-Scandinavian populations as well, its exact role in the pathogenesis of the disease remains to be determined. 
Table 1. Composition, Age, and Sex Distribution in Patients and Healthy Subjects

\begin{tabular}{|c|c|c|c|c|c|c|c|c|}
\hline \multicolumn{2}{|c|}{ Patients } & \multirow{2}{*}{$\begin{array}{c}\text { PEX } \\
(n)\end{array}$} & \multirow{2}{*}{$\begin{array}{c}\text { PEXG } \\
(n)\end{array}$} & \multicolumn{3}{|c|}{ Age } & \multirow[b]{2}{*}{ Female } & \multirow[b]{2}{*}{ Male } \\
\hline Origin & $n$ & & & Mean $\pm S D$ & Minimum & Maximum & & \\
\hline \multicolumn{9}{|l|}{ Patients } \\
\hline German & 517 & 206 & 311 & $76.6 \pm 8.51$ & 49 & 99 & 287 & 230 \\
\hline Italian & 209 & 76 & 133 & $78.3 \pm 7.72$ & 60 & 101 & 127 & 82 \\
\hline \multicolumn{9}{|l|}{ Controls } \\
\hline German & 348 & - & - & $73.9 \pm 6.4$ & 51 & 92 & 196 & 151 \\
\hline Italian & 70 & - & - & $75.2 \pm 7.37$ & 57 & 90 & 45 & 25 \\
\hline
\end{tabular}

Our study was designed to investigate association of three common LOXL1 polymorphisms with PEX and PEXG in two well-characterized patients' cohorts originating from Germany and Italy.

\section{Methods}

\section{Study Populations}

The study was approved by the ethics review boards of the Medical Faculty of the University of Erlangen-Nuremberg (Germany) and of the Monfalcone Hospital (Italy) and was in accordance with the tenets of the Declaration of Helsinki. All subjects gave informed consent before entering the study.

The group of 726 patients with PEX consisted of 517 subjects of German and 209 subjects of Italian origin (European). Exact composition, age, and sex distribution data of the two different patients' cohorts are reported below (Table 1). No significance difference regarding age and sex distribution between the groups of patients was noted. All German individuals underwent standardized clinical examinations for PEX at the Ophthalmologic Department of the University of Erlangen-Nuremberg (Erlangen, Germany), whereas all Italian patients were examined at the Ophthalmologic Department of the Hospital in Monfalcone (Italy) with identical clinical examinations. Unequivocal agreement was found between the clinical investigators CYM in Germany and DP in Italy. All patients recruited with PEX syndrome had to have manifest PEX material on the anterior capsule and pupillary margin in mydriasis, clearly visible on slitlamp biomicroscopy. Secondary open-angle glaucoma due to PEX syndrome was defined, if elevated intraocular pressure (IOP), an open chamber angle, characteristic visual field defects in computed perimetry and characteristic glaucomatous disc atrophy were found in the presence of manifest PEX deposits on the anterior lens capsule and/or pupillary margin.

A total of 418 healthy subjects were recruited. Three hundred forty-eight control individuals were of German and 70 were of Italian origin. Both groups were recruited from the same geographic regions as the patients, respectively. In addition, control subjects underwent ophthalmic examination and matched for age and sex (Table 1). Overall healthy individuals had IOP below $20 \mathrm{~mm} \mathrm{Hg}$, no glaucomatous disc damage, no PEX material deposits on anterior lens capsule and/or pupillary margin, no criteria indicating early or suspect PEX (e.g., atrophy of the iridal pigment epithelium at the pupillary margin, secondary melanin dispersion in the chamber angle and anterior chamber after dilation of the pupil, no dewlike condensation on the anterior, lens capsule, and normal mydriasis) and no family history of PEX and glaucoma. Visual acuity was at least 0.8 , and the optic media were clear for ophthalmic examination.

\section{DNA Extraction and Genotyping}

Genomic DNAs were extracted in the same laboratory from peripheral blood leukocytes of the 726 patients with PEX and 418 control individuals with automated techniques (AutoGenFlex 3000; AutoGen, Holliston, MA) using DNA chemistry (Flexigene; Qiagen, Hilden, Germany). SNP rs2165241 was genotyped with a predeveloped assay
(TaqMan; Applied Biosystems [ABI], Foster City, CA). Reactions were prepared according to manufacturer's instructions and performed on a sequence detection system (Prism 7900HT; ABI), by using standard thermal cycling conditions. The two nonsynonymous SNPs rs 3825942 and rs1048661 were genotyped through direct sequencing as the corresponding assays failed. Purified PCR products (AMPure; Agencourt Bioscience, Beverly MA, purified on a Biomek NX96 platform; Beckman Instruments, Fullerton, CA) were sequenced using dye termination chemistry (Prism Fluorescent Dye Termination; ABI). Purified sequence reactions (CleanSEQ; Agencourt Bioscience) were resolved on a sequence analyzer (3730xl Sequence Analyzer; ABI) and analyzed with genome assembly software (SEQMAN software; DNAStar, Madison, WI). The average genotyping rate was $98.5 \%$.

\section{Statistical Analysis}

Hardy-Weinberg equilibrium for all SNPs was confirmed in the case and control samples by using Haploview. ${ }^{16}$ Analysis of association by using allele counts and linkage disequilibrium-based haplotypes, was also performed with Haploview, which uses $\chi^{2}$ statistics for assessing haplotype association (ver. 3.2). ${ }^{16} P<0.05$ was considered statistically significant. Odds ratio (OR) and $95 \%$ confidence interval (CI) were calculated with opensource software written by D. J. R. Hutchon (http://www.hutchon.net/ConfidOR.htm). ${ }^{17}$

\section{Results}

Analysis of the single genotypes of the three LOXL1 common sequence variants $(\mathrm{rs} 2165241 \mathrm{~T}>\mathrm{C}$, $\mathrm{rs} 1048661 \mathrm{G}>\mathrm{T}$, and rs3825942G $>A$ ) in the PEX and PEXG patients from Germany and Italy, as well as respective control subjects are shown in Table 2 where differences in genotype distribution among patients and control subjects were detected in both populations. Strong association with the risk allele of each individual SNP (rs2165241T, rs1048661G, and rs3825942G) was observed in German samples of both PEX and PEXG, similar to that found in the Icelandic and Swedish samples ${ }^{12}$ with ORs between 2.49 and 3.26 (Table 3). Similar results were obtained in the Italian group with ORs ranging from 2.09 to 3.71 . No ORs could be calculated for rs3825942 (G153D), as all Italian patients analyzed were homozygous for the G allele at this SNP. Overall, allele frequencies in both populations in cases and control subjects were quite similar to those previously reported in Scandinavian patients. ${ }^{12}$ Thus, we combined the results from German and Italian patients groups and observed a maximum $\mathrm{OR}=2.43\left(P=2.90 \times 10^{-19}\right)$ for allele $\mathrm{G}$ of SNP rs1048661 and a maximum $\mathrm{OR}=4.87\left(P=8.22 \times 10^{-23}\right)$ for allele G of SNP rs3825942 (Table 3). It is also to note that, in both populations, the individual ORs of each single allele were similar for PEXG and PEX syndrome (Table 3) showing that the risk of glaucoma is due to the presence of PEX.

Haplotype analysis derived from allelic combination of the two nonsynonymous SNPs, rs1048661 and rs3825942, which are in complete linkage disequilibrium $\left(\mathrm{D}^{\prime}=1\right)$ detected only 
TABLE 2. Single Genotype Counts

\begin{tabular}{|c|c|c|c|c|c|c|c|c|c|}
\hline \multirow[b]{2}{*}{ Study Population ( $n$ ) } & \multicolumn{3}{|c|}{ rs2165241 } & \multicolumn{3}{|c|}{ rs1048661 } & \multicolumn{3}{|c|}{ rs3825942 } \\
\hline & $\mathrm{CC}$ & $\mathbf{C T}$ & TT & GG & GT & TT & GG & GA & $\mathbf{A A}$ \\
\hline \multicolumn{10}{|l|}{ Germany } \\
\hline Controls (348) & 98 & 158 & 84 & 136 & 165 & 41 & 250 & 78 & 18 \\
\hline PEXG (311) & 23 & 96 & 190 & 218 & 86 & 7 & 284 & 25 & 2 \\
\hline PEX (206) & 18 & 76 & 109 & 126 & 69 & 9 & 185 & 15 & 3 \\
\hline \multicolumn{10}{|l|}{ Italy } \\
\hline Controls (70) & 19 & 29 & 20 & 34 & 29 & 7 & 49 & 17 & 4 \\
\hline PEXG (133) & 3 & 47 & 82 & 84 & 44 & 2 & 130 & 0 & 0 \\
\hline $\operatorname{PEX}(76)$ & 2 & 26 & 45 & 53 & 22 & 1 & 76 & 0 & 0 \\
\hline
\end{tabular}

Genotype counts are provided for the three common sequence risk variants, rs2165241T $>\mathrm{C}$, rs1048661G $>\mathrm{T}$, and $\mathrm{rs} 3825942 \mathrm{G}>\mathrm{A}$, in German $(n=517)$ and Italian $(n=209)$ patients and respective controls subjects.

three of the four possible haplotypes (G-G; T-G, and G-A), both in the German and Italian groups (Table 4). Similar to the data from the Scandinavian population ${ }^{12}$ the haplotype (T-A) was not seen in our samples. Among the three haplotypes observed (G-G, T-G, and G-A), the main common haplotype in the population $(G-G)$ is the only overrepresented one in all patient groups of the two population and independent of the occurrence of glaucoma (Table 4). Thus, we calculated a combined OR for PEX and PEXG in German and Italian patients $(\mathrm{OR}=$ $\left.3.58 ; P=5.21 \times 10^{-43}\right)$. Moreover, two copies of the high-risk haplotype (G-G homozygosity) were identified in $66.1 \%$ of our total patients' cohorts compared with $27.3 \%$ of our complete control subjects $(\mathrm{OR}=5.2,95 \% \mathrm{CI}=3.99-6.78 ; P=1.7 \times$ $\left.10^{-37}\right)$. Our results clearly confirm the genetic association previously seen in Scandinavian populations.

\section{Discussion}

Our results convincingly show a genetic association of both PEX and PEXG with an intronic and two nonsynonymous coding SNPs of the LOXL1 gene as well as the haplotype encompassing the latter two in two independent European populations from Germany and Italy, confirming those initially detected in Scandinavian populations. ${ }^{12}$ A common haplotype (G-G) with a frequency of approximately $50 \%$ in control subjects is strongly associated with both PEX and PEXG in German and Italian populations and seems to be the strongest associated risk factor. Our findings indicate that this risk factor is common to several European populations, which is in agreement with the common disease-common haplotype hypothesis. ${ }^{18,19}$ In addition, as the frequency of the two risk alleles, as well as of the risk haplotype, in PEX cases without glaucoma is similar to that of PEX cases with glaucoma (Tables 3, 4) in both population, these data support the notion that these SNPs confer risk of glaucoma mainly through PEX.

The precise etiology and pathogenesis of PEX syndrome, however, remain poorly understood. Available immunohistochemical, biochemical, and molecular biological data strongly support the current concept that the fibrillar PEX deposits involve components of elastic fibers and microfibrils and that PEX syndrome is an elastic microfibrillopathy associated with an excessive production and aggregation of elastic microfibrils $^{6}$ or with an abnormal regulation of elastin synthesis. ${ }^{7,20,21}$

The functional significance of $L O X L 1$ in these PEX-associated elastotic processes is still unknown. To date apart from expression in lamina cribrosa cells and optic nerve head astrocytes, ${ }^{22}$ LOXL1 has been detected throughout the body in various organs, such as adult human lung, kidney, liver, heart, and muscle tissue, ${ }^{23,24}$ all of which are known to be affected by accumulations of PEX material. ${ }^{7}$ Recent studies have demonstrated that $L O X L 1$ is specifically targeted to sites of elastogenesis by binding of the LOXL1 propeptide to both tropoelastine

TABLe 3. ORs of LOXL1 Single-Risk Alleles at SNPS rs2165241 T, rs1048661 G, and rs3825942 G in PEX and PEXG from Germany and Italy

\begin{tabular}{|c|c|c|c|c|c|c|c|c|c|}
\hline \multirow[b]{2}{*}{ Study Groups $(n)$} & \multicolumn{3}{|c|}{ rs2165241 T } & \multicolumn{3}{|c|}{$\begin{array}{l}\text { rs1048661 G } \\
\text { LOXI1*141R }\end{array}$} & \multicolumn{3}{|c|}{$\begin{array}{l}\text { rs3825942 G } \\
\text { LOXL1*153G }^{*}{ }^{*} 1536\end{array}$} \\
\hline & Frq. & OR $(95 \% \mathrm{CI})$ & $\boldsymbol{P}$ & Frq. & OR $(95 \% \mathrm{CI})$ & $\boldsymbol{P}$ & Frq. & OR $(95 \% \mathrm{CI})$ & $\boldsymbol{P}$ \\
\hline \multicolumn{10}{|l|}{ Germany } \\
\hline Controls (348) & 0.482 & & & 0.644 & & & 0.857 & & \\
\hline PEX combined (517) & 0.752 & $3.26(2.65-4.01)$ & $6.77 \times 10^{-30}$ & 0.818 & $2.49(1.99-3.12)$ & $4.32 \times 10^{-16}$ & 0.951 & $3.26(2.28-4.66)$ & $1.21 \times 10^{-11}$ \\
\hline PEXG (311) & 0.770 & $3.60(2.83-4.58)$ & $1.74 \times 10^{-26}$ & 0.839 & $2.89(2.21-3.77)$ & $1.40 \times 10^{-15}$ & 0.953 & $3.41(2.22-5.24)$ & $4.78 \times 10^{-9}$ \\
\hline PEX (206) & 0.724 & $2.82(2.16-3.68)$ & $7.04 \times 10^{-15}$ & 0.787 & $2.04(1.54-2.71)$ & $7.08 \times 10^{-7}$ & 0.948 & $3.06(1.87-4.99)$ & $3.15 \times 10^{-6}$ \\
\hline \multicolumn{10}{|l|}{ Italy } \\
\hline Controls (70) & 0.515 & & & 0.693 & & & 0.821 & & \\
\hline PEX combined (209) & 0.798 & $3.71(2.44-5.63)$ & $2.19 \times 10^{-10}$ & 0.825 & $2.09(1.35-3.25)$ & 0.0009 & 1.000 & $\infty$ & $1.66 \times 10^{-18}$ \\
\hline PEXG (133) & 0.799 & $3.75(2.38-5.91)$ & $5.18 \times 10^{-9}$ & 0.815 & $1.96(1.22-3.15)$ & 0.0053 & 1.000 & $\infty$ & $1.96 \times 10^{-12}$ \\
\hline PEX (76) & 0.795 & $3.64(2.15-6.16)$ & $8.79 \times 10^{-7}$ & 0.842 & $2.36(1.34-x 4.16)$ & 0.0024 & 1.000 & $\infty$ & $5.08 \times 10^{-8}$ \\
\hline \multicolumn{10}{|l|}{ Combined } \\
\hline Controls (418) & 0.488 & & & 0.652 & & & 0.851 & & \\
\hline PEX combined (726) & 0.765 & $3.42(2.85-4.11)$ & $1.28 \times 10^{-40}$ & 0.820 & $2.43(2.00-2.97)$ & $2.90 \times 10^{-19}$ & 0.965 & $4.87(3.46-6.85)$ & $8.22 \times 10^{-23}$ \\
\hline PEXG (444) & 0.779 & $3.70(3.00-4.57)$ & $1.43 \times 10^{-35}$ & 0.832 & $2.64(2.10-3.32)$ & $1.89 \times 10^{-17}$ & 0.967 & $5.15(3.39-7.82)$ & $4.26 \times 10^{-17}$ \\
\hline PEX (282) & 0.743 & $3.03(2.40-3.84)$ & $5.90 \times 10^{-21}$ & 0.802 & $2.16(1.67-2.78)$ & $1.75 \times 10^{-9}$ & 0.962 & $4.48(2.78-7.21)$ & $3.02 \times 10^{-11}$ \\
\hline
\end{tabular}

SNP rs2165241 is located in the first intron of LOXL1, rs1048661 and rs3825942 cause amino-acid changes R141L and G153D, respectively. Frq., frequency; OR $\infty$, OR infinity. 
and fibulin- 5 and that these interactions are essential for directing the deposition of the enzyme onto elastic fibers. ${ }^{14}$ The two risks coding SNPs (rs1048661 and rs382542) are located in the $\mathrm{N}$-terminal part of pro-LOXL1 which was suggested to be critical in ensuring proper enzyme activation and in identifying the appropriate substrate that is to be acted on by the enzyme. ${ }^{14,15}$ As consequence these SNPs may influence targeting of proLOXL1 to tropoelastin or mediate the interaction of LOXL1 with other substrates

Analyses of adipose tissues have shown that the expression of $L O X L 1$ is decreased by $7.7 \%$ per risk allele of SNP rs 1048661 (R141L), ${ }^{12}$ which is a small change, but in a late-onset disease it could be relevant. It is notable, however, that the risk allele G of rs3825942 (G153D), the variant that confers the greater risk in all population studies so far and interestingly the only one present in the Italian patient cohort, has no effect on LOXL1 expression, at least in adipose tissues. Nevertheless, inadequate levels of LOXL1 in systemic and ocular tissues could predispose to an impaired elastin homeostasis and elastotic processes. Alternatively, sequence variations in the LOXL1 propeptide may alter the substrate specificity of LOXL1 and may lead to abnormal cross-linking, aggregation and insolubilization of elastic microfibrillar components into the typical PEX fibers.

Further studies correlating the genetic variants in the LOXL1 and ocular tissue changes associated with PEX are now needed to confirm the association of SNPs rs1048661 and rs382542 with this condition and to elucidate its functional consequences.

\section{Acknowledgments}

The authors thank all patients and healthy subjects for participating in the study; Juliane Niedziella for invaluable help with patient recruitment; and Claudia Preller for excellent technical assistance.

\section{References}

1. Ritch R, Schlotzer-Schrehardt U. Exfoliation syndrome. Surv Opbthalmol. 2001;45(4):265-315.

2. Bialasiewicz AA, Wali U, Shenoy R, Al-Saeidi R. Patients with secondary open-angle glaucoma in pseudoexfoliation (PEX) syndrome among a population with high prevalence of PEX: clinical findings and morphological and surgical characteristics (in German). Ophthalmologe. 2005;102(11):1064-1068.

3. Konstas AG, Stewart WC, Stroman GA, Sine CS. Clinical presentation and initial treatment patterns in patients with exfoliation glaucoma versus primary open-angle glaucoma. Opbthalmic Surg Lasers. 1997;28(2):111-117.

4. Ritch R, Schlotzer-Schrehardt U. Exfoliation (pseudoexfoliation) syndrome: toward a new understanding: proceedings of the First International Think Tank. Acta Opbthalmol Scand. 2001;79(2): 213-217.

5. Ovodenko B, Rostagno A, Neubert TA, et al. Proteomic analysis of exfoliation deposits. Invest Ophthalmol Vis Sci. 2007;48(4):14471457.

6. Schlotzer-Schrehardt U, Naumann GO. Ocular and systemic pseudoexfoliation syndrome. Am J Ophthalmol. 2006;141(5):921937.

7. Schlotzer-Schrehardt UM, Koca MR, Naumann GO, Volkholz H. Pseudoexfoliation syndrome: ocular manifestation of a systemic disorder? Arch Opbthalmol. 1992;110(12):1752-1756.

8. Ringvold A. Epidemiology of the pseudo-exfoliation syndrome. Acta Opbthalmol Scand. 1999;77(4):371-375.

9. Forsius H, Forsman E, Fellman J, Eriksson AW. Exfoliation syndrome: frequency, gender distribution and association with climatically induced alterations of the cornea and conjunctiva. Acta Opbthalmol Scand. 2002;80(5):478-484.

10. Damji KF, Bains HS, Stefansson E, et al. Is pseudoexfoliation syndrome inherited? - a review of genetic and nongenetic factors and a new observation. Opbthalmic Genet. 1998;19(4):175-185. 
11. Orr AC, Robitaille JM, Price PA, et al. Exfoliation syndrome: clinical and genetic features. Ophthalmic Genet. 2001;22(3):171-185.

12. Thorleifsson G, Magnusson KP, Sulem P, et al. Common sequence variants in the LOXL1 gene confer susceptibility to exfoliation glaucoma. Science. 2007;317(5843):1397-1400.

13. Kagan HM, Li W. Lysyl oxidase: properties, specificity, and biological roles inside and outside of the cell. J Cell Biochem. 2003;88(4): $660-672$.

14. Thomassin L, Werneck CC, Broekelmann TJ, et al. The pro-regions of lysyl oxidase and lysyl oxidase-like 1 are required for deposition onto elastic fibers. J Biol Chem. 2005;280(52):42848-42855.

15. Liu X, Zhao Y, Gao J, et al. Elastic fiber homeostasis requires lysyl oxidase-like 1 protein. Nat Genet. 2004;36(2):178-182.

16. Barrett JC, Fry B, Maller J, Daly MJ. Haploview: analysis and visualization of LD and haplotype maps. Bioinformatics. 2005;21(2): 263-265.

17. Bland JM, Altman DG. Statistics notes: the odds ratio. BMJ. 2000; 320(7247): 1468 .

18. Cardon LR, Abecasis GR. Using haplotype blocks to map human complex trait loci. Trends Genet. 2003;19(3):135-140.
19. Di Rienzo A, Hudson RR. An evolutionary framework for common diseases: the ancestral-susceptibility model. Trends Genet. 2005; 21(11):596-601.

20. Netland PA, Ye H, Streeten BW, Hernandez MR. Elastosis of the lamina cribrosa in pseudoexfoliation syndrome with glaucoma. Ophthalmology. 1995;102(6):878-886.

21. Pena JD, Netland PA, Vidal I, Dorr DA, Rasky A, Hernandez MR. Elastosis of the lamina cribrosa in glaucomatous optic neuropathy. Exp Eye Res. 1998;67(5):517-524.

22. Urban Z, Agapova O, Hucthagowder V, Yang P, Starcher BC, Hernandez MR. Population differences in elastin maturation in optic nerve head tissue and astrocytes. Invest Ophthalmol Vis Sci. 2007; 48(7):3209-3215.

23. Kenyon K, Modi WS, Contente S, Friedman RM. A novel human cDNA with a predicted protein similar to lysyl oxidase maps to chromosome 15q24-q25. J Biol Chem. 1993;268(25):1843518437.

24. Kim Y, Boyd CD, Csiszar K. A new gene with sequence and structural similarity to the gene encoding human lysyl oxidase. J Biol Chem. 1995;270(13):7176-7182. 